\title{
Self-scheduling of Wind-Thermal Systems Using a Stochastic MILP Approach
}

\author{
Rui Laia ${ }^{1,2}$, Isaias L.R. Gomes ${ }^{1,2}$, Hugo M.I. Pousinho ${ }^{1}$, Rui Melício ${ }^{1,2(\bowtie)}$, \\ and Victor M.F. Mendes ${ }^{2,3}$ \\ ${ }^{1}$ Instituto Superior Técnico, IDMEC, Universidade de Lisboa, Lisbon, Portugal \\ ruimelicio@gmail.com \\ 2 Departamento de Física, Universidade de Évora, Escola de Ciências e Tecnologia, \\ Évora, Portugal \\ ${ }^{3}$ Instituto Superior de Engenharia de Lisboa, Lisbon, Portugal
}

\begin{abstract}
In this work a stochastic (Stoc) mixed-integer linear programming (MILP) approach for the coordinated trading of a price-taker thermal (Ther) and wind power (WP) producer taking part in a day-ahead market (DAM) electricity market (EMar) is presented. Uncertainty (Uncer) on electricity price (EPr) and WP is considered through established scenarios. Thermal units (TU) are modelled by variable costs, start-up (ST-UP) technical operating constraints and costs, such as: forbidden operating zones, minimum (Min) up/down time limits and ramp up/ down limits. The goal is to obtain the optimal bidding strategy (OBS) and the maximization of profit (MPro). The wind-Ther coordinated configuration (CoConf) is modelled and compared with the unCoConf. The CoConf and unCoConf are compared and relevant conclusions are drawn from a case study.
\end{abstract}

Keywords: Wind thermal coordination $\cdot$ Stochastic programming $\cdot$ MILP

\section{Introduction}

The emissions derived of the use of nonrenewable fuels and the aspiration to attain independence of energy [1] lead a considerable European countries to promote generation of the electricity from renewable (Rnew) resources by adopting some instruments of support for Rnew energy production, namely investments incentives, green certificates, soft balancing costs and feed-in-tariffs [2].

At the end of 2014, 43.70\% of all novel Rnew farms were based on WP and was the 7 th year consecutively that over $55.0 \%$ of added capacity of power in the EU was Rnew [3]. In the face of the increasing Rnew energy incorporation in the last years, supply of energy still depending on nonrenewable fuels since more than $60 \%$ of the electricity generated all over the world in 2012 was based on nonrenewable fuel Ther plants [4].

In a restructured EMar, power resources owners' operate under competition level due to the nodal variations of EPr [5] in order to obtain the best revenue bidding in the DAM [6]. For the WP producers (WPP), WP and the market-clearing EPr Uncer are to be addressed in order to know the amount of energy to produce in order to present optimal 
offers. In absence of conformity, i.e., there is a deviation (Dev), economic penalizations is due to happen [7]. For Ther power producers, only market-clearing EPr Uncer has to be addressed.

\section{Relationship to Smart Systems}

A smart system can be stated as an embedded system that incorporates advanced systems and provide the inhabitants with sophisticated monitoring and control over how something happens in the system [8], for example a wind farm or a TU. Smart systems are capable of sensing, making diagnosis, describing, qualifying and managing how something happens in the system, incorporating both technical intelligence and cognitive functions. In smart systems, electronic devices will be communicating with software base system, allowing the user to access information about the functionality of the system [8]. These systems are highly reliable, often miniaturized, networked, predictive and energy autonomous [9]. Future power systems should ensure security, reliability and efficiency in energy management. Using the abilities of smart systems to monitoring the energy demand and the energy production of other units can play a vital role in what regards the unit commitment of TU. Particularly, monitoring and high quality real-time data of the exploitation of Rnew energy sources, namely WP, that usually requires a certain amount of spinning reserve due to their intermittent nature may represent additional information at the moment of unit commitment of TU. With this information, the Wind-Ther Power Producer (WTPP) can make a more accurate decision concerning the participation in EMars and therefore foremost revenue [10, 11]. Also, benefits of environmental are predictable with the increase in the capability of discovery offers able to be satisfied with a high level of being pleased and less needed of spinning reserve, less TU are needed and less nonrenewable fuel is used.

\section{State of the Art}

For Ther conversion of energy into electricity, several methods of optimization to resolve the problem of unit commitment (UC) have been used in the literature, including a technique of primacies list, classical mathematical programming techniques, like Lagrangian Relaxation (LR) and dynamic programming (DP) and more newly artificial intelligence (AI) techniques [12]. Although, requiring small computation time and easy to implement, the priority list technique does not guarantee an opportune resolution near the global optimal one, which implies an operation of higher cost [13, 14]. DP methods are flexible but these methods are characterized by a known limitation by the "curse of dimensionality". Although the LR can overcome the previous limitation, does not necessarily lead to a viable resolution, implying further processing for satisfying the infringed constraints in order to find a viable resolution, which does not guarantee solution optimal. Although, AI techniques based on simulating annealing and ANN have been applied, the major limitation of the AI techniques concerning with the possibility to obtain a resolution near the global optimum one is a disadvantage. The MILP method has been useful with success for solving the problem of UC [15]. MILP is suitable for 
the formulation of bidding strategies due to its rigorousness and extensive capability of modeling [16]. WPP usually have significant difficulties to predict their power output accurately. In addition, WPP have to face Uncer on EPr. These Uncer have to be expediently considered, i.e., treated into the variables of the problems [17] to be addressed by a WPP in order to know how much to produce and the price for bidding. The technical literature presents methods for WP bidding strategies solving using different approaches: the first one is the use of WP with technologies of storage of energy [18]; the use of economic options as a tool for WPP to hedge against WP Uncer [19]; another approach is the design of Stoc models in order to obtain OBS for WPP participating in an EMar [20], without the aforementioned policies. The 3rd line of action is a Stoc formulation explicitly modelling the Uncer faced by a WPP [21], using indeterminate measures and an established of scenarios built by WP forecast and market-clearing EPr forecast [22] requests.

Hence, this paper provides an effective approach based on Stoc MILP to find out the optimal bidding strategies of a single entity having to manage a coordinated wind-Ther system, so as to maximize the expected revenue in the Iberian day-ahead EMar.

\section{Problem Formulation}

\subsection{WPP}

Considering the variability and intermittent nature of WP the physical delivering usually differs from the offer submitted by WPP to the DAM. The revenue $R V_{h}$ of a WPP proposing a power of $E_{h}^{\text {offer }}$, but actually producing $E_{h}^{\text {act }}$ for period $h$ is stated as:

$$
R V_{h}=\pi_{h}^{D} E_{h}^{o f f e r}+I C_{h}
$$

In (1), the DAM price is $\pi_{h}^{D}$, the imbalance (Imb) cost is $I C_{h}$. The total Dev for period $h$ is stated as:

$$
D_{h}=E_{h}^{a c t}-E_{h}^{o f f e r}
$$

The price that WPP will pay for excess of production is $\pi_{h}^{+}$, the price to be charged for deficit of production is $\pi_{t}^{-}$. The Imb prices can be given by means of price ratios stated as:

$$
p r_{h}^{+}=\frac{\pi_{h}^{+}}{\pi_{h}^{D}}, p r_{h}^{+} \leq 1 \quad \text { and } \quad p r_{h}^{-}=\frac{\pi_{h}^{-}}{\pi_{h}^{D}}, p r_{h}^{-} \geq 1
$$

In (3), the $\pi_{h}^{+}$is never greater than 1 . The $\pi_{t}^{-}$is never lower than 1 .

The power producer

The operating cost, $T_{\text {si }}$, for a TU can is stated as:

$$
T_{s i h}=B_{i} b_{s i h}+g_{s i h}+u_{s i h}+A_{i} z_{s i h} \quad \forall s, \quad \forall i, \quad \forall h
$$


In (4), the fixed production cost is $B_{i}$, the added variable cost is $g_{s i h}$, the ST-UP and shut-down (Sh-Down) costs are $u_{s i h}$ and $A_{i}$, of the unit. The last three costs are in general described by nonlinear function (Func) and worse than that some of the functions are non-convex and non-differentiable functions, but some kind of smoothness is expected and required to use MILP, for instance, as being sub differentiable functions.

The ST-UP and Sh-Down costs of units in (4) are considered to be such that is possible to approximate those Func by a piecewise linear. Hence, the $g_{s i h}$, is:

$$
\begin{gathered}
g_{s i \mathrm{~h}}=\sum_{l=1}^{L} T_{i}^{l} \Delta_{s i \mathrm{~h}}^{l} \quad \forall s, \quad \forall i, \quad \forall h \\
E_{s i \mathrm{~h}}=E_{i}^{\min } b_{s \mathrm{~h} h}+\sum_{l=1}^{L} \Delta_{s i \mathrm{~h}}^{l} \quad \forall s, \quad \forall i, \quad \forall h \\
\left(M_{i}^{1}-E_{i}^{\min }\right) j_{s i \mathrm{~h}}^{1} \leq \Delta_{s i \mathrm{~h}}^{1} \quad \forall s, \quad \forall i, \quad \forall h \\
\Delta_{s i \mathrm{~h}}^{1} \leq\left(M_{i}^{1}-\mathrm{E}_{i}^{\min }\right) b_{s i \mathrm{~h}} \quad \forall s, \quad \forall i, \quad \forall h \\
\left(M_{i}^{l}-M_{i}^{l-1}\right) j_{s i \mathrm{~h}}^{l} \leq \Delta_{s i \mathrm{~h}}^{l} \quad \forall s, \quad \forall i, \quad \forall h, \quad \forall l=2, \ldots, L-1 \\
\Delta_{s i \mathrm{~h}}^{l} \leq\left(M_{i}^{l}-M_{i}^{l-1}\right) j_{s i \mathrm{~h}}^{l-1} \quad \forall s, \quad \forall i, \quad \forall h, \quad \forall l=2, \ldots, L-1 \\
0 \leq \Delta_{s i \mathrm{~h}}^{L} \leq\left(E_{i}^{\max }-M_{s i \mathrm{~h}}^{L-1}\right) j_{s i \mathrm{~h}}^{L-1}
\end{gathered}
$$

In (5), the slope of each segment is $T_{i}^{l}$, the segment power is $\Delta_{s i h}^{l}$. In (6), the binary variable $b_{\text {sih }}$ guarantee that the power production is equal to 0 if the unit is in the state offline. In (7), if the binary variable $j_{s i h}^{l}$ has a null value, then the segment power $\Delta_{s i h}^{1}$ can be lower than the segment 1 maximum power (MaxPow); otherwise and in conjunction with (8), if the unit is in the state on, then $\Delta_{s i h}^{1}$ is equal to the segment 1 MaxPow. In (9), if the binary variable $j_{s i h}^{l}$ has a null value, then the segment power $\Delta_{s i h}^{l}$ can be lower than the segment $l$ MaxPow; otherwise and in conjunction with (10), if the unit is in the state on, then $\Delta_{s i h}^{l}$ is equal to the segment $l$ MaxPow. The exponential nature of the ST-UP costs functions, $u_{s i h}$ is approached by a linear formulation [21] is:

$$
u_{s i \mathrm{~h}} \geq K_{i}^{\alpha}\left(b_{s i \mathrm{~h}}-\sum_{r=1}^{\alpha} b_{s i \mathrm{~h}-r}\right) \quad \forall s, \quad \forall i, \quad \forall h
$$

The constraints to limit the power produced by the unit are:

$$
\begin{gathered}
E_{i}^{\min } b_{s i h} \leq E_{s i \mathrm{~h}} \leq E_{s i h}^{\max } \quad \forall s, \quad \forall i, \quad \forall h \\
E_{s i h}^{\max } \leq E_{i}^{\max }\left(b_{s i h}-z_{s i h+1}\right)+S D z_{s i h+1} \quad \forall s, \quad \forall i, \quad \forall h
\end{gathered}
$$




$$
\begin{gathered}
E_{s i h}^{\max } \leq E_{s i \mathrm{~h}-1}^{\max }+R U b_{s i h-1}+S U y_{s i h} \quad \forall s, \quad \forall i, \quad \forall h \\
E_{s i \mathrm{~h}-1}-E_{s i \mathrm{~h}} \leq R D b_{s i \mathrm{~h}}+S D z_{s i \mathrm{~h}} \quad \forall s, \quad \forall i, \quad \forall h
\end{gathered}
$$

In (13) and (14), the upper bound of $E_{s i h}^{\max }$ is established, which is the maximum available power of the unit. The minimum down time (MDT) constraint is imposed by a formulation:

$$
\sum_{h=1}^{F_{i}} b_{s i \mathrm{~h}}=0 \quad \forall s, \quad \forall i
$$

$$
\begin{gathered}
\sum_{h=k}^{k+D H_{i}-1}\left(1-b_{s i \mathrm{~h}}\right) \geq D H_{i} z_{s i \mathrm{~h}} \quad \forall s, \quad \forall i, \quad \forall k=F_{i}+1 \ldots H-D H_{i}+1 \\
\sum_{h=k}^{H}\left(1-b_{s i \mathrm{~h}}-z_{s i \mathrm{~h}}\right) \geq 0 \quad \forall s, \quad \forall i, \quad \forall k=H-D H_{i}+2 \ldots H \\
F_{i}=\min \left\{H,\left(D H_{i}-t_{s i 0}\right)\left(1-b_{s i 0}\right)\right\}
\end{gathered}
$$

The MUT constraint is also imposed:

$$
\sum_{h=1}^{N_{i}}\left(1-b_{s i \mathrm{~h}}\right)=0 \quad \forall s, \quad \forall i
$$

$$
\begin{gathered}
\sum_{h=k}^{k+U H_{i}-1} b_{s i \mathrm{~h}} \geq U H_{i} y_{s i \mathrm{~h}} \quad \forall s, \quad \forall i, \quad \forall k=N_{i}+1 \ldots H-U H_{i}+1 \\
\sum_{h=k}^{H}\left(b_{s i \mathrm{~h}}-z_{s i \mathrm{~h}}\right) \geq 0 \quad \forall s, \quad \forall i, \quad \forall k=H-U H_{i}+2 \ldots H \\
N_{i}=\min \left\{H,\left(U H_{i}-U_{s i 0}\right) b_{s i 0}\right\}
\end{gathered}
$$

The relation between the binary variables to identify start-up, shutdown and forbidden operating zones is:

$$
\begin{gathered}
y_{s i \mathrm{~h}}-z_{s i \mathrm{~h}}=t_{s i \mathrm{~h}}-b_{s i \mathrm{~h}-1} \quad \forall s, \quad \forall i, \quad \forall h \\
y_{s i \mathrm{~h}}+z_{s i \mathrm{~h}} \leq 1 \quad \forall s, \quad \forall i, \quad \forall h
\end{gathered}
$$

The total power produced by the TU is:

$$
E_{s t}^{g}=\sum_{i=1}^{I} E_{s i \mathrm{~h}} \quad \forall s, \quad \forall h
$$


Objective function: The total offer is:

$$
E_{s h}^{o f f e r}=E_{s h}^{t h}+E_{s h}^{D} \quad \forall s, \quad \forall h
$$

The physical delivering is:

$$
E_{s h}^{a c t}=E_{s h}^{g}+E_{s h}^{\omega d} \quad \forall s, \quad \forall h
$$

In (27), $E_{s h}^{g}$ is the physical delivering by the TU and $E_{s h}^{\omega d}$ is the physical delivering by the wind farm for scenario $s$. The expected revenue of the GNCO is:

$$
\sum_{s=1}^{N_{s}} \sum_{h=1}^{N_{H}} p_{s}\left[\left(\pi_{s h}^{D} E_{s h}^{o f f e r}+\pi_{s h}^{D} p r_{s h}^{+} D_{s h}^{+}-\pi_{s h}^{D} p r_{s h}^{-} D_{s h}^{-}\right)-\sum_{i=1}^{I} T_{s i h}\right] \quad \forall s, \quad \forall h
$$

Subject to:

$$
\begin{gathered}
0 \leq E_{s h}^{\text {offer }} \leq E_{s h}^{M} \quad \forall s, \quad \forall h \\
D_{h s}=\left(E_{s h}^{a c t}-E_{s h}^{o f f e r}\right) \quad \forall s, \quad \forall h \\
D_{h s}=D_{h s}^{+}-D_{h s}^{-} \quad \forall s, \quad \forall h \\
0 \leq D_{h s}^{+} \leq E_{h s} x_{s} \quad \forall \omega, \quad \forall h
\end{gathered}
$$

The maximum Ther generation is:

$$
E_{s h}^{M}=\sum_{i=1}^{I} E_{s i \mathrm{~h}}^{\max }+E^{W \max } \quad \forall s, \quad \forall h
$$

An additional constraint for (28) appears:

$$
\left(E_{s \mathrm{~h}}^{\text {offer }}-E_{s^{\prime} \mathrm{h}}^{o f f e r}\right)\left(\pi_{s \mathrm{~h}}^{D}-\pi_{s^{\prime} \mathrm{h}}^{D}\right) \geq 0 \quad \forall s, s^{\prime}, \quad \forall h
$$

\section{Case Study}

The case study is from a GNCO with a WTPP, with 1440 MW of installed capacity. The used data is available in [6]. The energy prices are from the Iberic Market of electricity and available in [23], considering 10 days of June. The EPr and the energy generated from wind are displayed in Fig. 1. 

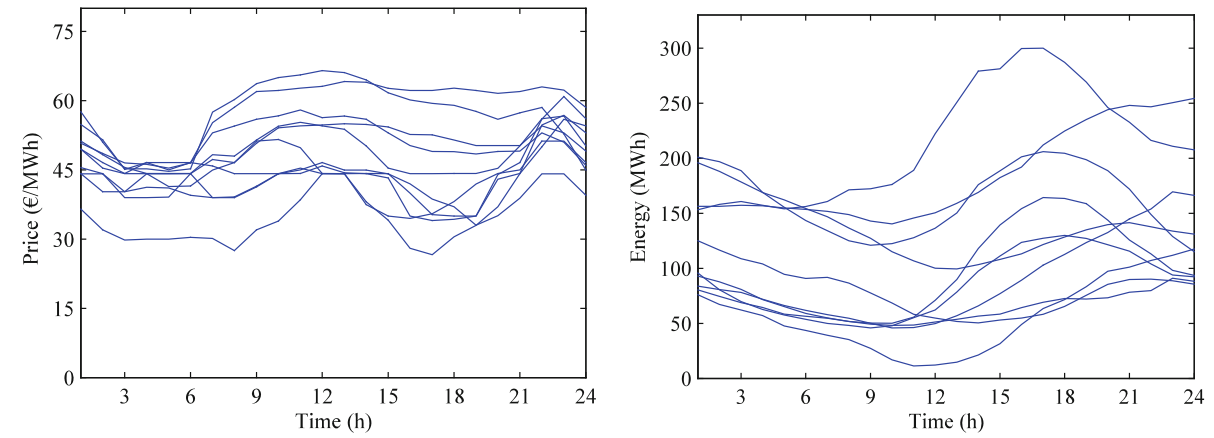

Fig. 1. Market Iberic: June 2014; left: EPr, energy from wind: right.

The energy generated is obtained using the total energy generated from the wind farm having $360 \mathrm{MW}$ of rated power. The expected revenue for CoConf and unCoConf are displayed in Table 1.

Table 1. Expected revenue for CoConf and uncoordinated configurations

\begin{tabular}{l|l}
\hline Case & Expected revenue \\
\hline Wind uncoordinated $(€)$ & 119200 \\
\hline Ther uncoordinated $(€)$ & 516848 \\
\hline Coordinated Wind and Ther $(€)$ & 642326 \\
\hline Gain $(\%)$ & 0,99
\end{tabular}

The non-decreasing energy bid for the unCoConf approach is displayed in Fig. 2.
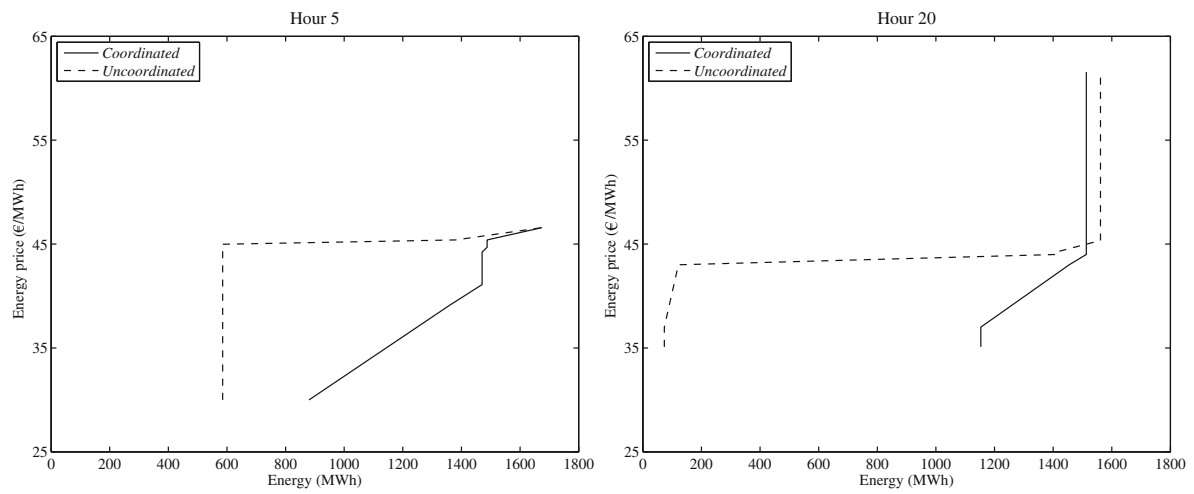

Fig. 2. Bids of energy.

In Fig. 2 the CoConf permits for a Min value of offered power upper than the one offered in the unCoConf and permits for a lesser price of the offering, which is a possible operation benefit. 


\section{Conclusion}

Smart Systems can play an important role for a Ther and WP producer since the operation till the bidding in day-ahead EMars. The ability to provide real-time data from the wind production may result in foremost decisions for the decision-maker and therefore higher revenues. As result of the proposed approach for uncoordinated and coordinated operations optimal schedule of the TU and the short-term bidding strategies are obtained. The presented approach is appropriate for the GNCO involvement with TU and a wind farm. The offer coordinated of Ther with WP power permits providing foremost outcomes than the sum of the lonely offers. The Uncer are modelled using established scenarios for the prices of the energy and power production. In the literature of all trading problems and management involving production by wind prove to be optimization problems under Uncer.

Acknowledgments. Portuguese Funds partially support this work through the FCT-Foundation for Science and Technology, LAETA 2015/2020, UID-EMS-50022-2013.

\section{References}

1. Al-Awami, A.T., El-Sharkawi, M.A.: Coordinated trading of wind and thermal energy. IEEE Trans. Sustain. Energy 2(3), 277-287 (2011)

2. Pineda, S., Bock, A.: Renewable-based generation expansion under a green certificate market. Renew. Energy 91, 53-63 (2016)

3. Wind in power: 2014 European Statistics, February 2015. http://www.ewea.org/fileadmin/ files/library/publications/statistics/EWEA-Annual-Statistics-2014.pdf

4. Key World Energy Statistics 2014: International Energy Agency (2014). http://www.iea.org/ newsroomandevents/agencyannouncements/key-world-energy-statistics-2014-now-availablefor-free.html

5. Laia, R., Pousinho, H.M.I., Melício, R., Mendes, V.M.F.: Bidding strategy of wind-thermal energy producers. Renew. Energy 99, 673-681 (2016)

6. Laia, R., Pousinho, H.M.I., Melício, R., Mendes, V.M.F.: Self-scheduling and bidding strategies of thermal units with stochastic emission constraints. Energy Convers. Manag. 89, 975-984 (2015)

7. Laia, R., Pousinho, H.M.I., Melício, R., Mendes, V.M.F.: Optimal scheduling of joint windthermal systems. Adv. Intell. Syst. Comput. 527, 136-146 (2017). Springer

8. Arsan, T.: Smart systems: from design to implementation of embedded smart systems. In: 13th International Symposium on Smart MicroGrids for Sustainable Energy Sources Enabled by Photonics and IoT Sensors, Nicosia, Cyprus, pp. 59-64 (2016)

9. Topham, D., Gouze, N.: Smart Systems Integration Knowledge Gateway. SSI, Copenhagen (2015)

10. Laia, R., Pousinho, H.M.I., Melício, R., Mendes, V.M.F.: Optimal bidding strategies of windthermal power producers. In: Camarinha-Matos, Luis M., Falcão, António J., Vafaei, N., Najdi, S. (eds.) DoCEIS 2016. IAICT, vol. 470, pp. 494-503. Springer, Cham (2016). doi: 10.1007/978-3-319-31165-4_46

11. Laia, R., Pousinho, H.M.I., Melício, R., Mendes, V.M.F.: Bidding decision of wind-thermal GenCo in day-ahead market. Energy Procedia 106, 87-96 (2016) 
12. Trivedi, A., Srinivasan, D., Biswas, S., Reindl, T.: Hybridizing genetic algorithm with differential evolution for solving the unit commitment scheduling problem. Swarm Evol. Comput. 23, 50-64 (2015)

13. Laia, R., Pousinho, H.M.I., Melício, R., Mendes, V.M.F.: Stochastic emission constraints on unit commitment. Procedia Technol. 17, 437-444 (2014)

14. Gomes, I.L.R., Pousinho, H.M.I., Melício, R., Mendes, V.M.F.: Bidding and optimization strategies for wind-pv systems in electricity markets assisted by CPS. Energy Procedia 106, 111-121 (2016)

15. Ostrowski, J., Anjos, M.F., Vannelli, A.: Tight mixed integer linear programming formulations for the unit commitment problem. IEEE Trans. Power Syst. 27(1), 39-46 (2012)

16. Floudas, C., Lin, X.: Mixed integer linear programming in process scheduling: modeling, algorithms, and applications. Annals of Operations Res. 139, 131-162 (2005)

17. El-Fouly, T.H.M., Zeineldin, H.H., El-Saadany, E.F., Salama, M.M.A.: Impact of wind generation control strategies, penetration level and installation location on electricity market prices. IET Renew. Power Gener. 2, 162-169 (2008)

18. Angarita, J.L., Usaola, J., Martinez-Crespo, J.: Combined hydro-wind generation bids in a pool-based electricity market. Electr. Power Syst. Res. 79(7), 1038-1046 (2009)

19. Hedman, K.W., Sheble, G.B.: Comparing hedging methods for wind power: using pumped storage hydro units vs options purchasing. In: Proceedings of International Conference on Probabilistic Methods Applied to Power Systems, Stockholm, Sweden, pp. 1-6 (2006)

20. Matevosyan, J., Soder, L.: Minimization of imbalance cost trading wind power on the shortterm power market. IEEE Trans. Power Syst. 21(3), 1396-1404 (2006)

21. Ruiz, P.A., Philbrick, C.R., Sauer, P.W.: Wind power day-ahead uncertainty management through stochastic unit commitment policies. In: Proceedings IEEE Power Systems Conference and Exposition, Seattle, USA, pp. 1-9 (2009)

22. Coelho, L.S., Santos, A.A.P.: A RBF neural network model with GARCH errors: application to electricity price forecasting. Electr. Power Syst. Res. 81(1), 74-83 (2011)

23. Red Electrica de España (2016). http://www.esios.ree.es/web-publica/ 